\title{
Synthesis of bicyclo[3.3.1]nonane derivatives containing fused heterocyclic rings
}

\author{
Linas Labanauskas, ${ }^{* 1,2}$ Albinas Zilinskas, ${ }^{1}$ Sigita Visniakova, ${ }^{1}$ Gintaras Urbelis, ${ }^{1,2}$ Olga \\ Gedrimaite, ${ }^{2}$ Ricardas Rozenbergas, ${ }^{2}$ Andrey Podgursky ${ }^{3}$ \\ ${ }^{1}$ Vilnius university, Naugarduko 24, LT-03225 Vilnius, Lithuania \\ ${ }^{2}$ Instutute of Biochemistry, Mokslininku 12, LT-08662 Vilnius, Lithuania \\ ${ }^{3}$ Zelinsky institute of Organic chemistry, Leninsky Prospect 47, 117913 Moscow, Russia \\ E-mail: linas.labanauskas@,chf.vu.lt
}

\begin{abstract}
The condensation reactions of 3,7-dihalobicyclo[3.3.1]nonan-2,6-dione or 3-bromobicyclo[3.3.1]nonan-2one (racemates) with 4-amino-2,4-dihydro-3H-triazolothione, 2-thiobenzimidazole and thiocarbamide were performed. New compounds containing bicyclo[3.3.1]nonane framework with fused thiazole, imidazothiazole and 1,8a-dihydro-5H-[1,2,4] triazolo[3,4- $b][1,3,4]$ thiadiazine rings were synthesized.
\end{abstract}

Keywords: 3,7-Dihalobicyclo[3.3.1]nonan-2,6-diones, 3-bromobicyclo[3.3.1]nonan-2-one, thiazole, imidazothiazole, 1,8a-dihydro-5H-[1,2,4]triazolo[3,4-b][1,3,4]thiadiazine

\section{Introduction}

Previously it was reported that compounds containing two properly allocated pyrimidine, indole and other rings in its structure possess self-assembling properties and can form supramolecular structures $^{1,2}$ as well as the natural compounds ${ }^{3}$. The presence of bicyclo[3.3.1]nonane core in synthetic compounds is necessary to keep two heterocyclic parts of molecule at such positions that they could form intermolecular hydrogen or electrostatic bonds which would support regular intermolecular structures. On the other hand, bicyclo[3.3.1]nonane derivatives with heterocyclic moieties are target compounds in re-synthesis of natural compounds ${ }^{4-6}$ and synthesis of their analogues (taxoids) used in drug research ${ }^{7,8}$. A series of new compounds containing two polar heterocyclic moyeties, fixed in a proper conformation by the framework of bicyclo[3.3.1]nonane and their analogues with one heterocyclic moiety were synthesized. 


\section{Results and Discussion}

Compounds 2-4 bearing two heterocyclic rings were synthesized by the condensation of 3,7dichlorbicyclo[3.3.1]nonan-2,6-dione (1) (racemate) ${ }^{9-11}$ with 4-amino-2,4-dihydro-3Htriazolothione $^{12}$, 2-thiobenzimidazole and thiocarbamide (Scheme 1).

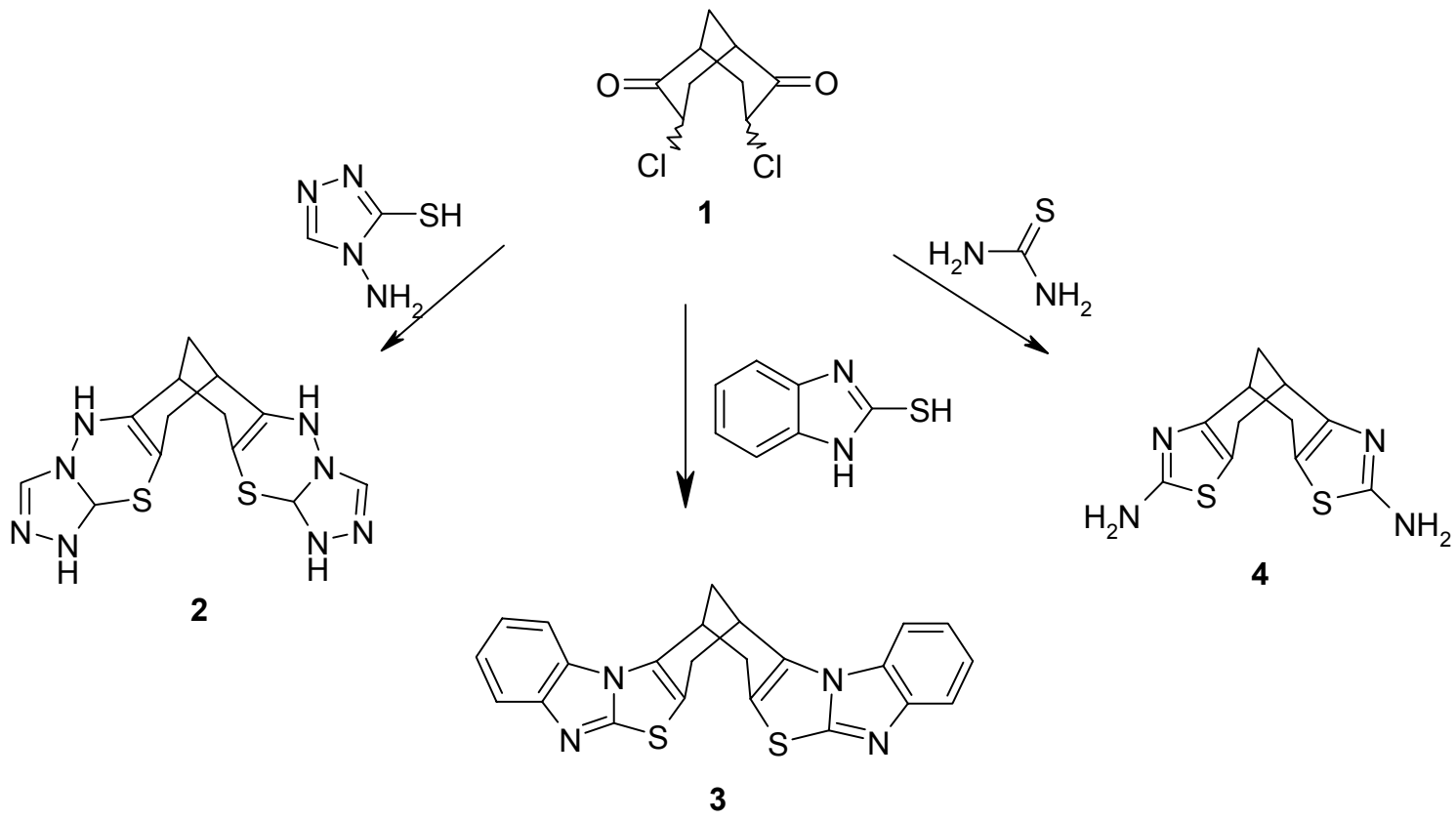

\section{Scheme 1}

The yields of products 2-4 has decreased by $10-20 \%$ in the cases if the dibromo analogue $e^{9-11}$ of compound 1 was used. The formation of bigger amounts of by-products in these cases was observed. Compounds 6-8 bearing one heterocyclic ring were synthesized analogously by the condensation of 3-bromobicyclo[3.3.1]nonan-2-one ${ }^{13}(5)$ (racemate) with 4-amino-2,4-dihydro$3 \mathrm{H}$-triazolothione, 2-thiobenzimidazole and thiocarbamide (Scheme 2). 


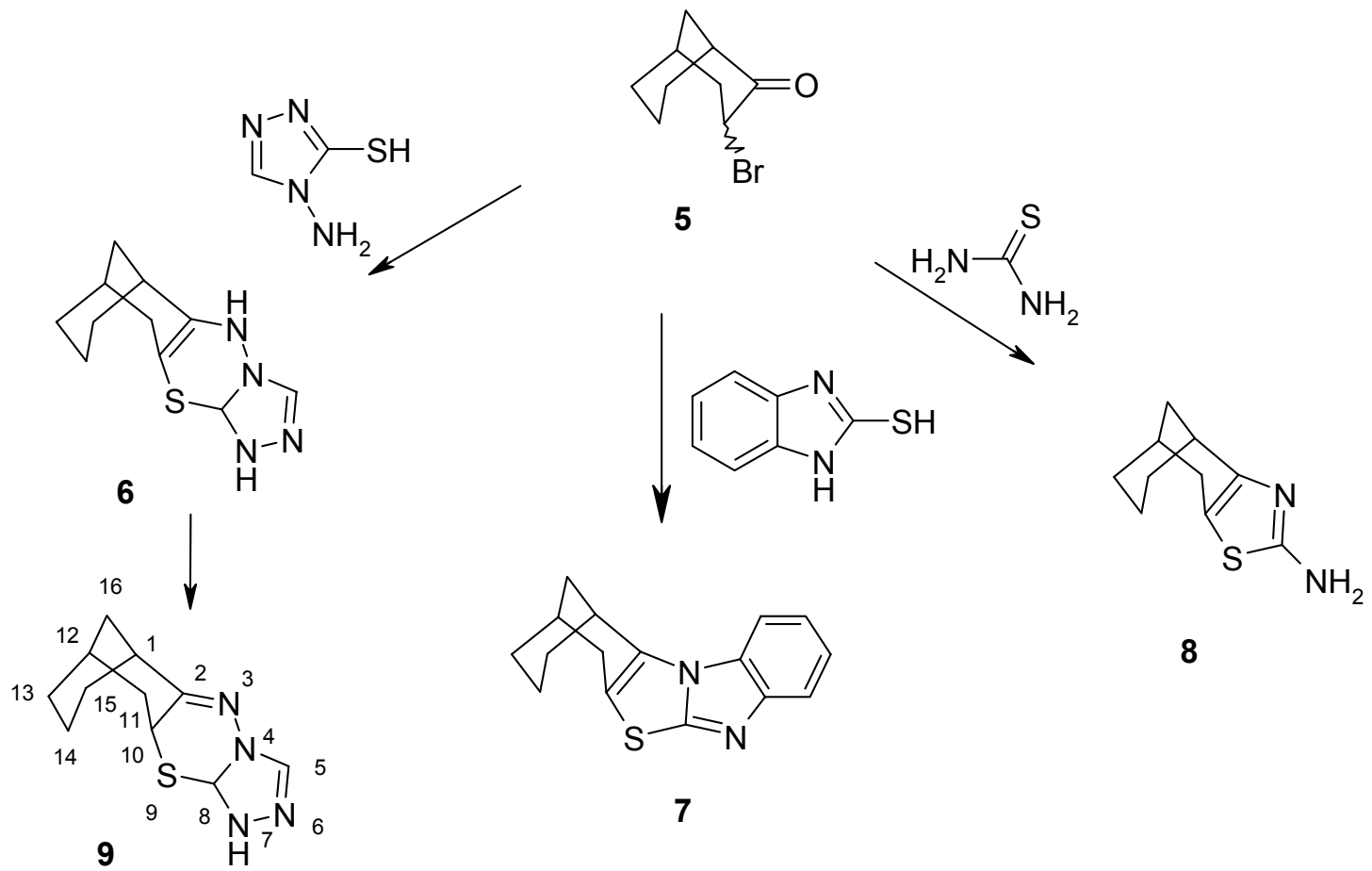

\section{Scheme 2}

Compounds 4 and 8 were synthesized in two steps by Hantzsh ${ }^{14}$. The first step of condensation was performed in ethanol by reflux for 3 hours. The second step required melting of reaction mixture without solvent. 2-Thiobenzimidazole reacted with alpha-haloketones $\mathbf{1 ,} 5$ and gave final condensation products under milder conditions. Thus, compounds $\mathbf{3}$ and $\mathbf{7}$ have been synthesized in ethanol solution by reflux within 3 hours. The reaction of 4-amino-2,4dihydro- $3 H$-triazolothione with $\mathbf{1}, \mathbf{5}$ in boiling ethanol was completed within 10 hours. Surprisingly, compound $\mathbf{6}$ has showed significant unstability. It has partially rearranged to $\mathbf{9}$ in several hours by standing.

The structures of compounds 2-4 and 6-9 were confirmed by comparison of experimental and calculated chemical shifts in ${ }^{1} \mathrm{H}$ and ${ }^{13} \mathrm{C}$ spectra. The means of chemical shifts were calculated using VAMP ${ }^{15}$ program using geometries of 3-9 optimized with the semi-empirical PM3 $\operatorname{method}^{16}$.

\section{Experimental Section}

General Procedures. Melting points were determined in open capillaries and are uncorrected. IR spectra were recorded in $\mathrm{KBr}$ on a Perkin-Elmer spectrophotometer model FT-IR Spectrum BX II. ${ }^{1} \mathrm{H}-\mathrm{NMR}$ spectra were recorded on Varian Unity Inova $(300 \mathrm{MHz})$. The purity of compounds was checked on silica gel coated plates (Merck). 
9,20-Dithio-3,4,6,7,14,15,17,18-octaazahexacyclo $\left[10.10 .10^{2,10} \cdot 0^{4,8} \cdot 0^{13,21} \cdot 0^{15,19}\right]$ tricosa-

2(10),5,13(21),16-tetraene (2). The solutions of $0.23 \mathrm{~g}(0.2 \mathrm{mmol})$ 4-amino-2,4-dihydro-3H1,2,4-triazol-3-thione in $10 \mathrm{~mL}$ ethanol and $0.221 \mathrm{~g} \quad(0.1 \mathrm{mmol})$ 3,7dichloromobicyclo[3.3.1]nonan-2,6-dione in $15 \mathrm{~mL}$ ethanol were joined, refluxed with stirring for $2 \mathrm{~h}$ and cooled to room temperature. Obtained crystalline product was filtered off to give $0.282 \mathrm{~g}(67 \%) 2$ (dihydrobromide), m. p. $>300{ }^{\circ} \mathrm{C}$. IR $\left(\mathrm{cm}^{-1}\right): 3429(\mathrm{~N}-\mathrm{H}), 1615$ and 1632 $(\mathrm{C}=\mathrm{C}), 1480$ and $1443(\mathrm{C}=\mathrm{N}) .{ }^{1} \mathrm{H}$ NMR $\left(\mathrm{CD}_{3} \mathrm{COOD}\right) \delta: 2.21-2.43\left(4 \mathrm{H}, \mathrm{m}, \mathrm{CH}_{2}\right), 2.57-2.65$ $(2 \mathrm{H}, \mathrm{m}, \mathrm{CH}), 3.40\left(4 \mathrm{H}\right.$, br. s, $\left.\mathrm{CH}_{2}\right), 3.49$ (4H, br. s, $\left.\mathrm{CH}_{2}\right), 8.82(\mathrm{~N}=\mathrm{C}-\mathrm{H}, 2 \mathrm{H}, \mathrm{s}), 10.12$ (s, N-H, $\mathrm{HBr}$ ). Anal. Calcd. for $\mathrm{C}_{13} \mathrm{H}_{18} \mathrm{Cl}_{2} \mathrm{~N}_{8} \mathrm{~S}_{2}$ (421.37): C, 37.05\%; H, 4.31\%; Cl, 16.83\%; N, 26.59\%; S, $15.22 \%$; Found: C, 36.87\%; H, 4.47\%; N, 26.85\%

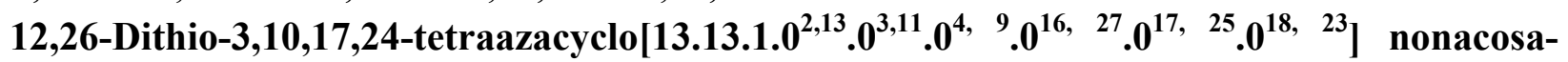
2(13),4,6,8,10,16(27),18,20,22,24-decaene (3). The solutions of $0.22 \mathrm{mg}$ (0.1mol) 3bromobicyclo[3.3.1]nonan-2-one in $10 \mathrm{~mL}$ ethanol and $0.33 \mathrm{~g}(0.22 \mathrm{mmol})$ 2-thiobenzimidazole in $10 \mathrm{~mL}$ ethanol were joined and refluxed with stirring for $3 \mathrm{~h}$. Solvent was evaporated, oily product was triturated with $10 \% \mathrm{NaOH}$ and washed with $\mathrm{H}_{2} \mathrm{O}$, dissolved in $\mathrm{CHCl}_{3}$, dried with $\mathrm{Na}_{2} \mathrm{SO}_{4}$ and fractionated on silica gel column using EtOAc to give $0.198 \mathrm{~g}(48 \%) 7$. IR (cm $\left.{ }^{-1}\right)$ : $1632(\mathrm{C}=\mathrm{C}) .{ }^{1} \mathrm{H}$ NMR $\left(\mathrm{CD}_{3} \mathrm{COOD}\right) \delta: 1.92$ - $1.95\left(2 \mathrm{H}, \mathrm{m}, \mathrm{CH}_{2}\right), 2.02$ - $2.06\left(2 \mathrm{H}, \mathrm{m}, \mathrm{CH}_{2}\right), 3.14$ $3.19\left(2 \mathrm{H}, \mathrm{m}, \mathrm{CH}_{2}\right), 3.61$ - $3.66(2 \mathrm{H}, \mathrm{m}, \mathrm{CH}), 7.43-7.46$ (4H, m, ArH), 7.73 - 7.76 (4H, m, ArH). Anal. Calcd. for $\mathrm{C}_{23} \mathrm{H}_{16} \mathrm{~N}_{4} \mathrm{~S}_{2}$ (412.54): C, 66.96\%; H, 3.91\%; N, 13.58\%; S, 15.54\%; Found: C, $67.14 \% ; \mathrm{H}, 4.08 \% ; \mathrm{N}, 13.66 \%$

\section{5,12-Dithio-3,10-diazatetracyclo $\left[6.6 .1 .0^{2,6} \cdot 0^{9,13}\right]$ pentadeca-2(6),3,9(13),10-tetraen-4,11-}

diamine (4). The solutions of $0.22 \mathrm{~g}(0.1 \mathrm{mmol})$ 3,7-dichlorbicyclo[3.3.1]nonan-2,6-dione in $20 \mathrm{~mL}$ ethanol and $0.18 \mathrm{~g}(0.24 \mathrm{mmol})$ thiocarbamide in $20 \mathrm{~mL}$ ethanol were joined and refluxed with stirring for $3 \mathrm{~h}$. The solvent was evaporated and the residue was kept at $180-200{ }^{\circ} \mathrm{C}$ for $1 \mathrm{~h}$. Obtained product was recrystallized from ethanol to give $0.25 \mathrm{~g}(75 \%) 4$ (dihydrochloride); $\mathrm{m}$. p. $300-303{ }^{\circ} \mathrm{C}$. IR $\left(\mathrm{cm}^{-1}\right): 3180,3120,3090,3030\left(\mathrm{NH}_{2}\right), 1632(\mathrm{C}=\mathrm{C}), 1583(\mathrm{C}=\mathrm{N}) .{ }^{1} \mathrm{H}$ NMR $\left(\mathrm{CD}_{3} \mathrm{OD}\right) \delta: 1.96\left(\mathrm{CH}_{2}, 2 \mathrm{H}, \mathrm{s}\right), 2.51-2.56\left(\mathrm{CH}_{2}, 2 \mathrm{H}, \mathrm{m}\right), 2.62\left(4 \mathrm{H}, \mathrm{d}, \mathrm{J}=16 \mathrm{~Hz}, \mathrm{CH}_{2}\right), 2.84(4 \mathrm{H}$, $\mathrm{dd}, \mathrm{J}=16$ and $4 \mathrm{~Hz}, \mathrm{CH}_{2}$,), 3.26- $3.36(\mathrm{CH}, 2 \mathrm{H}, \mathrm{m}) .{ }^{13} \mathrm{C} \mathrm{NMR}\left(\mathrm{CDCl}_{3}\right) \delta: 19.2$ (1-C), $19.5(8-\mathrm{C})$, 20.5 (7-C), 20.7 (14-C), 28.6 (16-C), 136.9 (6- and 13-C), 170.8 (2- and 9-C), 177.9 (4- and 11 C). Anal. Calcd. for $\mathrm{C}_{11} \mathrm{H}_{14} \mathrm{Cl}_{2} \mathrm{~N}_{4} \mathrm{~S}_{2}$ (337.29): C, 39.17\%; H, 4.18\%; Cl, 21.02\%; N, 16.61\%; S, 19.01\%; Found: C, 39.06\%; H, 4.34\%; N, 16.37\%.

9-Thio-3,4,6,7-tetraazatetracyclo $\left[10.3 .1 .0^{2,10} \cdot 0^{4,8}\right]$ hexadeca-2(10),5-diene (6). The solutions of $0.22 \mathrm{mg}(0.1 \mathrm{~mol}) 3$-bromobicyclo[3.3.1]nonan-2-one in $10 \mathrm{~mL}$ ethanol and $0.127 \mathrm{~g}(0.11 \mathrm{mmol})$ 4-amino-2,4-dihydro-3H-1,2,4-triazol-3-thion in $10 \mathrm{~mL}$ ethanol were joined and refluxed with stirring for $10 \mathrm{~h}$, cooled, neutralized with $10 \% \mathrm{Na}_{2} \mathrm{CO}_{3}$, evaporated, dissolved in $\mathrm{CHCl}_{3}$, dried with $\mathrm{Na}_{2} \mathrm{SO}_{4}$ and fractionated in silica gel column using the mixture of $\mathrm{CHCl}_{3}$ and diethyl ether in ratio 5:1 to give $0.114 \mathrm{~g}(48 \%)$ 6. IR $\left(\mathrm{cm}^{-1}\right): 3449(\mathrm{~N}-\mathrm{H}), 1638(\mathrm{C}=\mathrm{C}), 1483$ and $1445(\mathrm{C}=\mathrm{N})$. ${ }^{1} \mathrm{H} \mathrm{NMR}\left(\mathrm{CDCl}_{3}\right) \delta: 1.87$ - $2.07\left(6 \mathrm{H}, \mathrm{m} \mathrm{CH}_{2}\right), 2.36\left(2 \mathrm{H}, \mathrm{d}, \mathrm{J}=5 \mathrm{~Hz}, \mathrm{CH}_{2}\right), 2.67-2.79(2 \mathrm{H}, \mathrm{m}$, $\left.\mathrm{CH}_{2}\right), 2.98$ - $3.12(1 \mathrm{H}, \mathrm{m}, \mathrm{CH}), 4.05-4.08(1 \mathrm{H}, \mathrm{m}, \mathrm{CH}), 7.30(\mathrm{~N}-\mathrm{CH}, 2 \mathrm{H}, \mathrm{s}), 8.48(2 \mathrm{H}, \mathrm{s}, \mathrm{NH})$. ${ }^{13} \mathrm{C} \mathrm{NMR}\left(\mathrm{CDCl}_{3}\right) \delta$ : 18.7 (14-C), 21.8 (12-C), 26.0 (1-C), 31.2 (15-C), 31.4 (11-C), 33.3 (13-C), 
36.3 (16-C), 76.7 (8-C), 142.1 (5-C), 142.4 (10-C), 166.3 (2-C). Anal. Calcd. for $\mathrm{C}_{11} \mathrm{H}_{16} \mathrm{~N}_{4} \mathrm{~S}$ (236.34): C, 55.90\%; H, 6.82\%; N, 23.71\%; S, 13.57\%; Found: C, 56.02\%; H, 7.01\%; N, $23.69 \%$

12-Thio-3,10-diazapentacyclo[13.3.1.0 $\left.0^{2,13} \cdot 0^{3,11} \cdot 0^{4,9}\right]$ nonadeca-2(13),4,6,8,10-pentaene (7). The solutions of $0.22 \mathrm{mg}(0.1 \mathrm{~mol}) 3$-bromobicyclo[3.3.1]nonan-2-one in $10 \mathrm{~mL}$ ethanol and $0.165 \mathrm{~g}(0.11 \mathrm{mmol})$ 2-thiobenzimidazole in $10 \mathrm{~mL}$ ethanol were joined and refluxed with stirring for $4 \mathrm{~h}$. Solvent was evaporated, solid product was triturated with $10 \% \mathrm{NaOH}$ and washed with $\mathrm{H}_{2} \mathrm{O}$. Obtained oil was dissolved in $\mathrm{CHCl}_{3}$, dried with $\mathrm{Na}_{2} \mathrm{SO}_{4}$ and fractionated in silica gel column using the mixture of $\mathrm{CHCl}_{3}$ and $\mathrm{MeOH}$ in ratio $10: 1$ to give $0.175 \mathrm{~g}(65 \%) 7$. IR $\left(\mathrm{cm}^{-1}\right): 3138,3054\left(\mathrm{NH}_{2}\right), 1630(\mathrm{C}=\mathrm{C}), 1468(\mathrm{C}=\mathrm{N}) .{ }^{1} \mathrm{H}$ NMR $\left(\mathrm{CDCl}_{3}\right) \delta: 1.44-1.49\left(\mathrm{CH}_{2}\right.$, $2 \mathrm{H}, \mathrm{m}), 1.74-1.78\left(2 \mathrm{H}, \mathrm{m}, \mathrm{CH}_{2}\right), 1.79-1.83\left(2 \mathrm{H}, \mathrm{m}, \mathrm{CH}_{2}\right), 1.97-2.04\left(2 \mathrm{H}, \mathrm{m}, \mathrm{CH}_{2}\right), 2.40-$ $2.49\left(1 \mathrm{H}, \mathrm{m}, \mathrm{CH}_{2}\right), 2.58\left(1 \mathrm{H}, \mathrm{d}, \mathrm{J}=16 \mathrm{~Hz}, \mathrm{CH}_{2}\right), 3.08(1 \mathrm{H}, \mathrm{dd}, \mathrm{J}=16$ and $5 \mathrm{~Hz}, \mathrm{CH}), 3.62-3.66$ $(1 \mathrm{H}, \mathrm{m}, \mathrm{CH}), 7.08-7.18(2 \mathrm{H}, \mathrm{m}, \mathrm{ArH}), 7.64(1 \mathrm{H}, \mathrm{d}, \mathrm{J}=9 \mathrm{~Hz}, \mathrm{ArH}), 7.82(1 \mathrm{H}, \mathrm{d}, \mathrm{J}=9 \mathrm{~Hz}, \mathrm{ArH})$. ${ }^{13} \mathrm{C}$ NMR $\left(\mathrm{CDCl}_{3}\right)$ \&: 27.1 (17-C), 27.7 (18-C), 27.8 (14-C), 29.5 (1-C), 30.9 (15-C), 32.1 (19-C), 33.1 (16-C), 110.6 (5-C), 120.5 (13-C), 121.05 (8-C), 122,1 (6-C), 123.1 (7-C), 129.8 (4-C), 130.2 (2-C), 147.8 (9-C), 156.5 (11-C). Anal. Calcd. for $\mathrm{C}_{16} \mathrm{H}_{16} \mathrm{~N}_{2} \mathrm{~S}$ (268.38): C, 71.61\%; H, $6.01 \%$; N, 10.44\%; S, 11.95\%; Found: C, 50.16\%; H, 4.34\%; N, 20.97\%

5-Thio-3-azatricyclo[6.3.1.0 ${ }^{2,6}$ ]dodeca-2(6),3-dien-4-amine (8). The solutions of $0.1 \mathrm{~g}$ (0.46 mmol) 3-bromo-bicyclo[3.3.1]-nonan-2-one in $10 \mathrm{~mL}$ ethanol and $0.038 \mathrm{~g}(0.51 \mathrm{mmol})$ thiocarbamide in $10 \mathrm{~mL}$ ethanol were joined and refluxed with stirring for $4 \mathrm{~h}$. The solvent was evaporated and the residue was kept at $180-200{ }^{\circ} \mathrm{C}$ for $1 \mathrm{~h}$. Obtained product was triturated with $10 \% \mathrm{NaOH}$, washed with $\mathrm{H}_{2} \mathrm{O}$, dried and fractionated in silica gel column using ethyl acetate to give $0.05 \mathrm{~g}(56 \%) 8$. IR $\left(\mathrm{cm}^{-1}\right)$ : IR $\left(\mathrm{cm}^{-1}\right): 3135,3140\left(\mathrm{NH}_{2}\right), 1642(\mathrm{C}=\mathrm{C}), 1578(\mathrm{C}=\mathrm{N}) \cdot{ }^{1} \mathrm{H}$ NMR $\left(\mathrm{CDCl}_{3}\right) \delta: 0.98$ - $1.01\left(4 \mathrm{H}, \mathrm{m}, \mathrm{CH}_{2}\right), 1.18-1.40(1 \mathrm{H}, \mathrm{m}, \mathrm{CH}), 1.69-1.82\left(2 \mathrm{H}, \mathrm{m}, \mathrm{CH}_{2}\right)$, $2.25-2.38\left(\mathrm{CH}_{2}, 2 \mathrm{H}, \mathrm{m}\right), 2.41-2.52(1 \mathrm{H}, \mathrm{m}, \mathrm{CH}), 2.97-3.05\left(\mathrm{CH}_{2}, 2 \mathrm{H}, \mathrm{m}\right), 3.60(1 \mathrm{H}$, br. s, $\left.\mathrm{NH}_{2}\right), 5.21\left(1 \mathrm{H}\right.$, br. s, $\left.\mathrm{NH}_{2}\right)$. Anal. Calcd. for $\mathrm{C}_{10} \mathrm{H}_{14} \mathrm{~N}_{2} \mathrm{~S}(194.30)$ : C, 61.82\%; H, 7.26\%; N, $14.42 \%$; S, 16.50\%; Found: C, 62.03\%; H, 7.31\%; N, 14.28\%

9-Thio-3,4,6,7-tetraazatetracyclo $\left[10.3 .1 .0^{2,10} \cdot 0^{4,8}\right]$ hexadeca-2,5-diene (9) was formed from 6 and isolated by chromatography on silica gel column using $\mathrm{CHCl}_{3}$. IR $\left(\mathrm{cm}^{-1}\right): 3449(\mathrm{~N}-\mathrm{H}), 1638$ $(\mathrm{C}=\mathrm{C}), 1445(\mathrm{C}=\mathrm{N}), 1483(\mathrm{C}=\mathrm{N}) .{ }^{1} \mathrm{H}$ NMR $\left(\mathrm{CDCl}_{3}\right) \delta: 1.31-1.68\left(\mathrm{~m}, \mathrm{CH}_{2}, 2 \mathrm{H}\right), 1.70-1.84$ $\left(8 \mathrm{H}, \mathrm{m}, \mathrm{CH}_{2}\right), 2.19-2.23(1 \mathrm{H}, \mathrm{m}, \mathrm{CH}), 2.45-2.49(1 \mathrm{H}, \mathrm{m}, \mathrm{CH}), 3.49-3.52(1 \mathrm{H}, \mathrm{m}, \mathrm{CH}), 7.3$ $(\mathrm{N}-\mathrm{CH}, 2 \mathrm{H}, \mathrm{s}), 8.48(\mathrm{NH}, 1 \mathrm{H}, \mathrm{s}) .{ }^{13} \mathrm{C}$ NMR $\left(\mathrm{CDCl}_{3}\right)$ ) 28.3 (14-C), 29.9 (15-C), 37.2 (1-C), 33.1 (11-C), 33.7 (13-C), 34.1 (16-C), 36.1 (12-C), 38.4 (10-C), 77.5 (8-C), 142.5 (5-C), 164.6 (2-C). Anal. Calcd. for $\mathrm{C}_{11} \mathrm{H}_{16} \mathrm{~N}_{4} \mathrm{~S}$ (236.34): C, 55.90\%; H, 6.82\%; N, 23.71\%; S, 13.57\%; Found: C, $55.74 \%$; H, 6.63\%; N, 23.55\% 


\section{References}

1. Stončius, S.; Butkus, E.; Žilinskas, A.; Larsson, K.; Öhrström, L.; Berg, U.; Wärnmark, K. J. Org. Chem. 2004, 69, 5196.

2. Zilinskas, A; Labanauskas, L. Chem. Heterocycl. Comp. 2007, 7, 936.

3. Lehn, J.-M. PNAS 2002, 99, 4763.

4. Mehta, G.; Bera, M. K. Tetrahedron Lett. 2006, 47, 689.

5. Abe, M.; Nakada, M. Tetrahedron Lett. 2007, 48, 4873.

6. Abe, M.; Nakada, M. Tetrahedron Lett. 2006, 47, 6347.

7. Kingston, D. G. I.; Newman D. J. Current Opinion in Drug discovery \& Development, 2007, $10,130$.

8. Ganesh, T.; Norris, A. et al, Bioorganic \& Medicinal Chemistry, 2006, 14, 3447.

9. Butkus, E.; Žilinskas, A.; Kadziauskas, P.; Kubilius. R. Russ. Chem. Bull. 1997, 2, 328.

10. Butkus, E.; Kubilius, R.; Stončius, S.; Žilinskas, A. J. Chem. Soc., Perkin Trans. 1 1999, 1431.

11. Kubilius, R.; Stončius, A.; Žilinskas, A; Butkus, E. Chemija 1999, 319.

12. Beyer, M.; Kroper, C. F. Lieb. Ann. 1960, 637, 135.

13. Klusacek, H.; Musso, H. Chem. Ber. 1970, 103, 3066.

14. Hantzsch, A.; Weber, J. H. Chem. Ber. 1887, 20, 3118.

15. Clark, T.; Alex, B.; Chandrasekhar, P.; Gedeck, J; Horn, A.; Hutter, M.; Martin, B.; Rauhut, W.; Sauer, G; Schindler, T.; Steinke, T. VAMP, Version 7.5a, Universität Erlangen: Erlangen, 1999.

16. Stewart, J. J. P. J. Comput. Chem. 1989, 10, 2009. 